\title{
Pro-active Monitoring of Electronic Contracts
}

\author{
Lai Xu and Manfred A. Jeusfeld \\ Tilburg University, CRISM/Infolab \\ 5000 LE, Tilburg, The Netherlands \\ $\{$ L.Xu, Manfred.Jeusfeld $\}$ Ouvt.nl \\ http://infolab.uvt.nl/people/\{lxu,jeusfeld $\}$
}

\begin{abstract}
Contracts between multiple business partners play an increasing role in a global economy where activities along the value chain are executed by independent yet co-operating companies. Information technology to enact a value chain is now being deployed in the form of ERP systems and Web services. However, there is little known how to formally check whether such an enactment is indeed fulfilling the contract between the parties. In this paper, we investigate which parts of a contract can be formalized to be automatically monitored. Our approach not only supports the detection of actual violations but also pro-active detection of imminent contract violations.
\end{abstract}

\section{Introduction}

A contract between multiple business partners contains some statements about their business relationship, in particular on their physical and informational actions. One purpose of such a contract is to distinguish expected and acceptable behavior from forbidden behavior.

The introduction of workflow systems and enterprise resource planning systems increases the automation of business contract execution. To the same degree, the demand for automated monitoring increases because more information about the contract execution has to be processed by the business partners.

The business partners are interested in answers to the following questions:

1 Given the current state of contract execution, which actions are expected from a partner in the future.

2 Is a contract violation imminent, i.e. likely to happen within short time? Which partner has to be reminded to fulfill her obligation?

3 Which partner is responsible for a contract violation?

We address the problem by regarding it as a formalization problem: Given a paper contract, formalize it into suitable representations such that the three main questions can be answered. Essentially, we map informal requirements (the paper contract) into formal specifications that are subject to automated processing very much like system requirements are mapped into implementations. Due to lack of space, the paper shall put some emphasis on question 2, the pro-active monitoring of imminent contract violations. 
The paper uses a standard case study from the CrossFlow project 30 . throughout the paper (see Section 2 for more details). Section 3 presents the formal contract model and defines key concepts like actions (any activity mentioned in a contract), commitments (a sequence of activities promised by some partner to become true in the future), and contract constraints (a statement about the well-formedness of a contract execution). The latter are used to inform business partners about which actions are expected from them in the future. The commitments are sequences of action types which some contract partner is responsible to make true during contract execution.

Finally, Section 15 presents related work and the paper concludes.

\section{Case Study}

The paper describes a standard multi-partner scenario 30 which outlines the manner in which a car damage claim is handled by an insurance company (AGFIL). The contract parties work together to provide a service level which facilitates efficient claim settlement. The parties involved are called Europ Assist, Lee Consulting Services, Garages and Assessors. Europ Assist offers a 24-hour emergency call answering service to policyholders. Lee C.S. coordinates and manages the operation of the emergency service on a day-to-day level on behalf of AGFIL. Garages are responsible for car repair. Assessors conduct the physical inspections of damaged vehicles and agree repair upon figures with the garages.

The general process of a car insurance case is described as following: the policyholder phones Euro Assist using a free-phone number to notify a new claim. Euro Assist will register the information, suggest an appropriate garage, and notify AGFIL which will check whether the policy is valid and covers this claim. After AGFIL receives this claim, AGFIL sends the claim details to Lee C.S.. AGFIL will send a letter to the policyholder for a completed claim form. Lee C.S. will agree upon repair costs if an assessor is not required for small damages, otherwise an assessor will be assigned. The assessor will check the damaged vehicle and agree upon repair costs with the garage. After receiving an agreement of repairing car from Lee C.S., the garage will then commence repairs. After finishing repairs, the garage will issue an invoice to the Lee C.S., which will check the invoice against the original estimate. Lee C.S. returns all invoices to AGFIL. AGFIL processes the payment. In the whole process, if the claim is found invalid, all contractual parties will be contacted and the process will be stopped. The case study shows a rather complex workflow between multiple partners.

\section{Monitorable Contract Model}

A contract is an agreement between two or more parties that is binding to those parties and that is based on mutual commitments [12]. Our monitorable contract model (in Figure 1) consists of two core components: the Monitorable Element (ME) and the Monitoring Mechanism (MM) [20]. The ME includes the description of the trade process, specifically the actions and commitments involved. 
A commitment in this paper defined as a guarantee by one party towards other parties that some action sequence shall be executed completely provided that some trigger, involve and finish action happens, and the other parties fulfill their end of the bargain. ME also includes contract constraints for describing logic relationships among actions and guards of contract constraints for specifying the right order of actions which checks what obligations remain to be realized after the occurrence of the guarded action. The $\mathbf{M M}$ is a dynamic mechanism for contract monitoring, it consists of the monitoring module and the reactive module. The monitoring module includes the commitment graph, the maintaining guard algorithm and pro-active detection algorithm. A commitment graph is an overview of commitments between partners, so a commitment graph is the encoding of some contract clauses. The maintaining guard algorithm and pro-active detection algorithm dynamically trace which actions have occurred, which have not occurred but are expected to occur, and which may never occur, it provides a possibility for reminding and warning messages to be sent before anomalous actions occur. The reactive module has four submodules: reminding, warning, tracing, and compensating module. They respectively support anticipation and avoidance before anomalous actions occur, detection and compensation after anomalous actions occur. The $\mathbf{M E}$ and $\mathbf{M M}$ will be explained in the next sections.

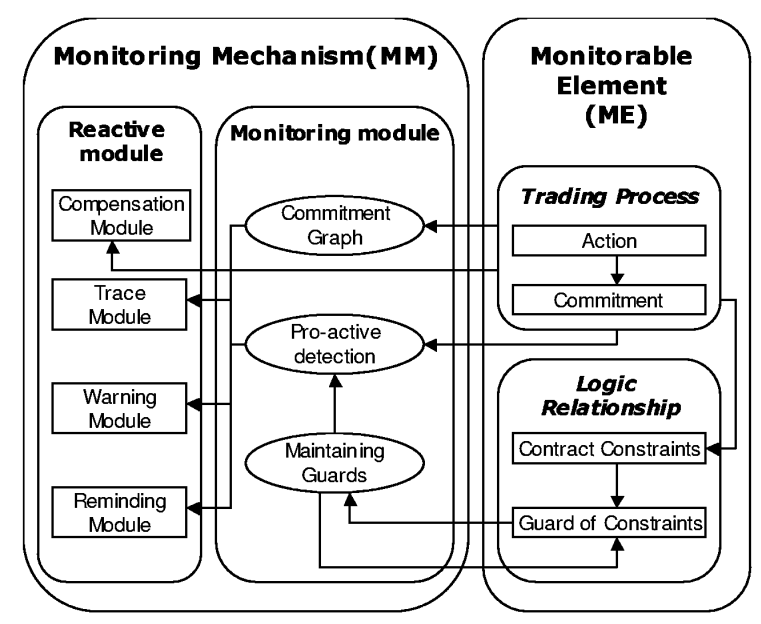

Fig. 1. The monitorable contract model

\subsection{The Monitorable Element}

Based on the car insurance case, actions and commitments between business partners are identified and specified. Contract constraints will be explained using all specified actions. Guards for each contract constraint scheme are calculated in following sections. 
Actions. An action is an atom in our contract model. An action is specified by an action name, a sender of the action, a receiver of the action and a deadline before which the action need to perform. Because a contract party can be involved in different commitments and play the different roles, we specify the roles of a party as $\mathcal{R}$. A set of total roles of a contract denotes as $\mathbb{R}$.

Definition 1 A party can act as the different roles in different commitments. Let $I D$ be a domain $I D$, roles of a party $\mathcal{R}_{x}$ can be specified as:

$$
\mathcal{R}_{x}=\left\{r_{1}, r_{2}, \ldots: r_{1}, r_{2}, \ldots \in I D\right\}
$$

Let $\mathcal{P}$ be a set of parties, the set of all roles is

$$
\mathbb{R}=\bigcup_{\forall x \in \mathcal{P}} \mathcal{R}_{x}
$$

Example 2 In car insurance, we use following abbreviations for different contractual parties: $P$ for Policyholders; AG for AGFIL; E for Europ Assist; L for Lee Consulting Services; $G$ for Garage; and A for Assessor. All partiers is a set $\mathcal{P}$ :

$$
\mathcal{P}=\{P, A G, E, L, G, A\}
$$

the garage $(G)$ involved to the repair service commitment, it acts as a repairer as role $G^{\prime}$. In the daily service commitment, the garage is a cooperator with Lee C.S. as role $G^{\prime \prime}$. In the pay repair cost commitment, the garage acts as a receiver as role $G^{\prime \prime \prime}$. Hence the garage plays three roles $G^{\prime}, G^{\prime \prime}, G^{\prime \prime \prime}$.

All the roles of the garage can act as is a set $\mathcal{R}_{\text {garage: }}$

$$
\mathcal{R}_{\text {garage }}=\left\{G^{\prime}, G^{\prime \prime}, G^{\prime \prime \prime}\right\}
$$

In car insurance case, a set of all roles $\mathbb{R}$ can be specified as:

$$
\mathbb{R}=\left\{P^{\prime}, P^{\prime \prime}, E, A G, L, A, G^{\prime}, G^{\prime \prime}, G^{\prime \prime \prime}\right\}
$$

An action has a particular name from domain ID, a sender of the action and a receiver of the action from $\mathbb{R}$ and a performance deadline of the action from time $\mathcal{T}$. Deadlines provide the flexibility to initiate actions at a more optimal time, rather than immediately invoking operations the moment a condition occurs.

Definition 3 Let $\mathbb{R}$ be a set of all roles of all parties, ID be the domain ID, and $\mathcal{T}$ be the time. An action is specified as:

$$
\text { action }=(\text { name }, \text { sender }, \text { receiver }, t)
$$

where name $\in I D$, sender, receiver $\in \mathbb{R}$ and $t \in \mathcal{T}$. We require all names of actions to be unique so they can be used as identifiers.

a set of actions $\mathbb{A}$ for a contract can be specified as :

$$
\mathbb{A}=\bigcup_{\forall x \in \mathcal{P}}\{\text { action }\}
$$

For car insurance case, all actions are specified in Appendix 2 of our research report 21]. According to contracts between partners, each action should support certain commitments. In next section, we specify commitments which are the key part of contracts. 
Commitments. In this paper, a commitment is a guarantee by one party towards other parties that some action sequence shall be executed completely provided that some trigger, involve, and finish action happens, and the other parties fulfill their end of the bargain. The notion of commitment in this paper is not related to beliefs, desires, or intentions [17 31. In Cohen and Levesque's research, commitments are related to establishing common beliefs about a certain state of the world [31. In our monitoring model, we do not reason about beliefs of the contractual parties involved, which Daskalopulu did in evidencebased contract performance monitoring 7]; we do not argue assessment of legal status and directives in business process automation which Abrahams did in [1]. The notion of commitment used in this paper is sufficient for the purpose for which it is used.

In a multi-party contract, for finishing a commitment, different party's actions are involved. Those actions can trigger, involve, and finish the commitment. For example, in the car repair service commitment, the garage first needs to receive the policyholder's car as a trigger of this commitment. The garage will repair the car after Lee C.S. agreed the repair cost which the garage estimated before. Actions included in a commitment have different attributes. A commitment is described by a commitment name, sender of the commitment, receiver of the commitment, and a series of actions and their attributes.

Definition 4 Let $\mathbb{A}$ be a set of actions, for each action a $\in \mathbb{A}$ can trigger(tr), involve(in) or finish (fi) a commitment, hence, each action has its attribute, attributes of actions $\mathcal{U}$ can be specified as:

$$
\mathcal{U}=\{t r, i n, f i\}
$$

Let ID be the domain ID, $\mathbb{A}$ be a set of actions, $\mathcal{P}$ be a set of parties and $\mathbb{R}$ be a set of all roles of all parties played. A commitment is specified as :

commitment $=\left(\right.$ name, sender, receiver, $\left.\left\{\left(a_{1}, u_{1}\right),\left(a_{2}, u_{2}\right), \ldots,\left(a_{n}, u_{n}\right): a_{i} \in \mathbb{A}, u_{i} \in \mathcal{U}\right\}\right)$

where name is an identifer, name $\in I D$; sender and receiver are the contract parties, sender, receiver $\in \mathbb{R} ; a_{1}, a_{2}, \ldots, a_{n}$ denotes all actions involved in the commitment and their attributes $u_{1}, u_{2}, \ldots, u_{n}$. We require all names of commitments to be unique so as they can be used as identifiers.

A set of commitments $\mathbb{M}$ can be specified as:

$$
\mathbb{M}=\bigcup_{\forall x \in \mathcal{P}}\{\text { commitment }\}
$$

Let $X=\{1,2,3, \ldots\}$ and $a \in \mathbb{A}$, a sequence function $f_{\text {position }}: \mathbb{A} \times \mathbb{M} \rightarrow X$,

$f_{\text {position }}\left(a_{i}, m\right)= \begin{cases}i & \text { iff } i \text { is the sequence number of action } a_{i} \text { in commitment } m \\ \text { undef } \text { otherwise }\end{cases}$ $f_{\text {position }}\left(a_{i}, m\right)$ is the position of action $a_{i}$ in the commitment $m$.

Examples of commitments of the car insurance case can be found in Appendix 3 of our research report [21]. 
Contract constraints. Among actions there exist logic relationships which we call contract constraints. Contract constraints show the occurrence order among actions in a business process ([29] and 28]). The formal specification of contract constraints is described as following,

Constraint 5 Using Temporal Logic (in Appendix 1 of our research report[21]), let $\mathbb{A}$ be a set of actions, and $a, b, c \in \mathbb{A}, \bar{a}, \bar{b}$ and $\bar{c}$ denotes $a, b$, and $c$ will never occur respectively. A contract constraint is defined using the follow schemes:

$$
\begin{aligned}
\bar{a} \wedge \bar{b} \vee a \cdot b & \text { (scheme 1) } \\
((\bar{a} \vee \bar{b}) \wedge \bar{c}) \vee a \cdot b \cdot c \vee b \cdot a \cdot c & \text { (scheme 2) } \\
a \cdot b \vee \bar{b} & \text { (scheme 3) } \\
a \cdot b \cdot c \vee b \cdot a \cdot c \vee \bar{c} & \text { (scheme 4) }
\end{aligned}
$$

Constraint scheme 1 denotes an initiation relationship, $a$ and $b$ can never occur, or $a$ and $b$ occur in order, which means $a$ must occur before $b$. Constraint scheme 2 captures a joint initiation relationship, if $a$ or $b$ never occur, $c$ will certainly never occur ; if $c$ occurs, $a$ and $b$ both have occurred before. Constraint scheme 3 describes an enable relationship, if $b$ occurs, that means $a$ has occurred before, otherwise $b$ will never occur. Constraint scheme 4 indicates a joint enable relationship; if $c$ occurs, $a$ and $b$ have occurred before, no matter with the order of $a$ and $b$ 's occurrence, otherwise c will never occur. The contract constraint schemes can be extended in different business applications. Examples of contract constraints can be found in Appendix 4 of our research report 21].

Guards of contract constraints. For each contract constraint scheme, the guard captures how far that scheme has progressed. After the occurrence of the guarded action, it checks what obligations remain to be realized. The guard of an action for a contract constraint regards to which actions have occurred, which have not occurred but are expected to occur, and which should not occur in the future.

Definition 6 Let $\mathcal{L}$ be a temporal logic language (see Appedix 1), some used symbols $-, \neg, \diamond, \square$ and $\cdot$ are introduced as following: $\bar{a}$ denotes a will never occur; $\neg$ a denotes a has not yet occurred; $\diamond a$ denotes a will eventually occur; $\square a$ denotes a has occurred; $a \cdot b$ denotes a will occur before $b . \mathcal{W}$ be a set of contract constraints, $\mathcal{S}$ be a constraint scheme in $\mathcal{W}, \mathcal{S} \in \mathcal{W}$, and $\mathbb{A}$ be a set of actions. $I_{1}, I_{2} \in \mathcal{S}, a_{1}, \ldots, a_{n} \in \mathbb{A}, \Gamma=\{a, \bar{a}: a \in \mathbb{A}\}$ is an alphabet, $\Gamma \subseteq \mathcal{L}$. A function $\mathrm{G}: \mathcal{S} \times \Gamma \mapsto \mathcal{L}$ is the guard of contract constraints. It is inductively defined as:

$$
\begin{aligned}
& \mathrm{G}\left(I_{1} \vee I_{2}, a\right) \equiv \mathrm{G}\left(I_{1}, a\right) \vee \mathrm{G}\left(I_{2}, a\right) \\
& \mathrm{G}\left(I_{1} \wedge I_{2}, a\right) \equiv \mathrm{G}\left(I_{1}, a\right) \wedge \mathrm{G}\left(I_{2}, a\right)
\end{aligned}
$$

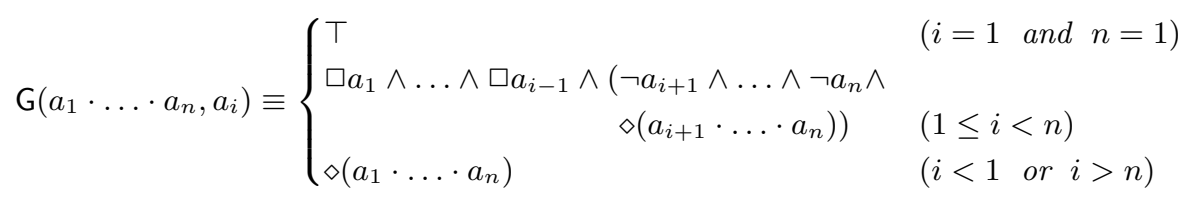




$$
\begin{aligned}
& \mathrm{G}\left(\overline{a_{1}} \cdot \ldots \cdot \overline{a_{n}}, a_{i}\right) \equiv \begin{cases}0 & (1 \leq i \leq n) \\
\diamond\left(\overline{a_{1}} \cdot \ldots \cdot \overline{a_{n}}\right) & (i<1 \text { or } i>n)\end{cases} \\
& \mathrm{G}\left(a_{1} \cdot \ldots \cdot a_{n}, \bar{a}_{i}\right) \equiv \begin{cases}0 & (1 \leq i \leq n) \\
\diamond\left(a_{1} \cdot \ldots \cdot a_{n}\right) & (i<1, \text { or } i>n)\end{cases} \\
& \mathrm{G}\left(\overline{a_{1}} \cdot \ldots \cdot \overline{a_{n}}, \bar{a}_{i}\right) \equiv \begin{cases}\top & (i=1 \text { and } n=1) \\
\square \overline{a_{1}} \wedge \ldots \wedge \square \overline{a_{i-1}} \wedge\left(\neg \overline{a_{i+1}} \wedge \ldots \wedge \neg \overline{a_{n}} \wedge\right. & (1 \leq i<n) \\
\diamond\left(\overline{a_{1}} \cdot \ldots \cdot \overline{a_{n}}\right) & (i<1, \text { or } i>n)\end{cases}
\end{aligned}
$$

$\mathrm{G}(0, a) \equiv 0$

$\mathrm{G}(\top, a) \equiv \top$

Example 7 Let $\mathbb{A}$ be a set of actions, $a, b \in \mathbb{A}, \Gamma$ be an alphabet, $a, \bar{a}, b, \bar{b} \in \Gamma$, guards of constraint scheme 1 (initiate scheme) as follows:

$$
\begin{aligned}
\mathrm{G}(\bar{a} \wedge \bar{b} \vee a \cdot b, a) & \equiv \mathrm{G}(\bar{a}, a) \wedge \mathrm{G}(\bar{b}, a) \vee \mathrm{G}(a \cdot b, a) \\
& \equiv(0 \wedge \diamond \bar{b}) \vee \neg b \wedge \diamond b \\
& \equiv \neg b \wedge \diamond b \\
\mathrm{G}(\bar{a} \wedge \bar{b} \vee a \cdot b, \bar{a}) & \equiv \diamond \bar{b} \\
\mathrm{G}(\bar{a} \wedge \bar{b} \vee a \cdot b, b) & \equiv \square a \\
\mathrm{G}(\bar{a} \wedge \bar{b} \vee a \cdot b, \bar{b}) & \equiv \diamond \bar{a}
\end{aligned}
$$

The guards of contract constraint scheme 1 mean: action $a$ can occur when $b$ has not yet occurred and may occur in the future; action $a$ can never occur if $b$ can never occur; $b$ can occur only if $a$ has occurred; $b$ will never occur when $a$ may never occur as well.

We explain the meaning of guards. According to example in Appendix 4 (1) of our research report[21, for equation Example 7(1), when Europ Assist has notified AGIFL about the claim, AGFIL has not yet sent a claim form to the policyholder, but AGFIL may send a claim form soon. For equation Example 7(2), if Europ Assist never send any claim to AGFIL, AGFIL may never send a claim form to the policyholder neither. For equation Example 73(3), if AGFIL has sent the claim to the policyholder, it means Europ Assist has sent this claim to AGFIL. For equation Example 7(4), if AGFIL never sends the claim to the policyholder, it means Europ Assist never sends a claim to AGFIL.

\subsection{The Monitoring Mechanism}

Our monitoring mechanism includes a monitoring module and a reactive module. The monitoring module observes occurred activities and captures any relevant information that arises during the fulfillment of the contract. The reactive module receives the triggered information based on the output of the monitoring modules and the monitorable contract, and sends a relevant message (such as 
warning and reminding) as responses. In the following sections, we will discuss the commitment graph, maintaining guard algorithm and pro-active detection algorithm in the monitoring module respectively.

Commitment graphs. It is widely accepted that commitments are the core of contracts, commitments are an even more important concept to specify multiparty contracts. In this section, we present a commitment graph which shows complex relationships among commitments. Commitment relationships are not only about a condition commitment relationship [22], for example, if a contractee first ships goods to a contractor, the contractor will pay to cost of goods later, the commitment of shipping goods is a condition to activate a commitment of payment. In our car insurance case, the relationship between repair service commitment and daily service commitment is a mixed relationship: after Lee C.S. agreed the repair cost in daily service commitment, the garage can repair the car in repair service commitment; after the garage repairs the car and returns the invoice, daily service commitment will go on to execute its following actions. In Figure 2 presents the commitment graph for the car in-

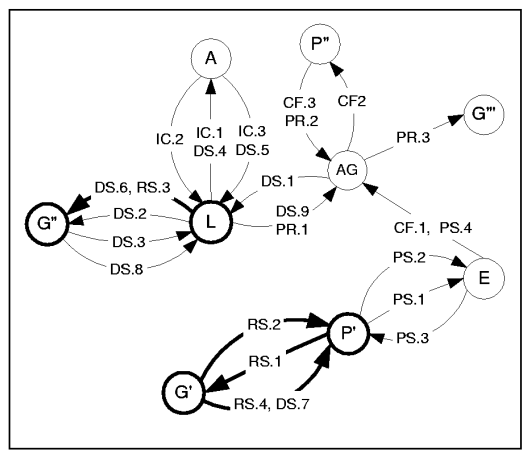

(a) Highlight repair service commitment C_repairService

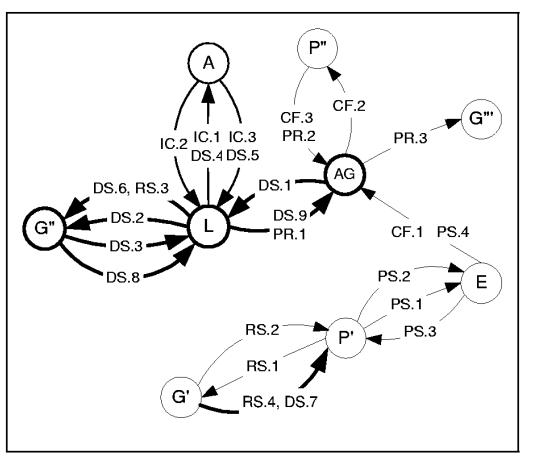

(b) Highlight daily service commitment C_dailyService

Fig. 2. Commitment Graph

surance case. For all edges of this graph, we use the following abbreviations: $P S$ for C_phoneService, $R S$ for C_repairService, $D S$ for C_dailyService, $I C$ for C_inspectCar, $P R$ for C_payRepairCost, $C F$ for C_claimForm. In Appendix 5 of our research report 21, we summarize commitments, involved actions and their attributes and abbreviations of each action.

\footnotetext{
${ }^{1}$ We regard commitment orders as an integral of contracts. They shall formalized in the Definition 9 (page 10).
} 
We know commitment C_repairService and commitment C_dailyService involve following actions from Appendix 3 of our research report 21].

(C_repairService, G, P, \{(A_sendCar, tr), (A_estimateRepairCost, $f i$ ),

$$
\text { (A_agreeRepairCar }, t r),(\text { A_repairCar }, f i)\})
$$

(C_dailyService, L, AG, \{(A_forwardClaim, tr), (A_contactGarage, in),

(A_sendRepairCost, in), (A_assignAssessor, in),

(A_sendNewRepairCost, $t r$ ), (A_agreeRepairCar, $f i$ ),

(A_repairCar, $t r),($ A_sendlnvoices, $i n)$,

(A_forwardlnvoices, $f i)\}$ )

We observe action A_agreeRepairCar includes in commitment C_repairService as the 3rd action and includes in commitment C_dailyService as the 6 th action. Action A_repairCar includes in commitment C_repairService as the 4th action also includes in commitment C_dailyService as the 7 th action. Hence, each action is indicated as an edge in a commitment graph, information about which commitment this action is involved in and the sequence of this action for a particular commitment is recorded as edge codes. An edge for action A_agreeRepairCar has two codes: C_repairService.3 and C_dailyService.6. In our commitment graph, we use edge code $R S$ for C_repairService and edge code $D S$ for C_dailyService, $R S .3$ and DS.6 both denote action A_agreeRepairCar. In Appendix 5 of our research report 21], we summarize commitments, involved actions and their attributes and abbreviations of each action.

Another example, the relationship between daily service commitment and inspect car commitment is an embedded relationship, if the repair cost is higher than a certain amount, daily service commitment will trigger inspect car commitment. After inspecting the car, the new repair cost is sent by a assessor and daily service commitment can continue. From Appendix 3 of our research report [21, we have:

(C_dailyService, L, AG, $\{($ A_forwardClaim, tr), (A_contactGarage, in),

(A_sendRepairCost, in), (A_assignAssessor, in),

(A_sendNewRepairCost, tr), (A_agreeRepairCar, $f i$ ),

(A_repairCar, $t r)$, (A_sendlnvoices, $i n)$,

(A_forwardlnvoices, $f i)\}$ )

(C_inspectCar, A, L, $\{($ A_assignAssessor,$t r),($ A_inspectCar, in ),

(A_sendNewRepairCost,$f i)\}$ )

After all actions include in commitment C_inspectCar are finished, commitment C_dailyService can continue. We use $I C$ for C_inspectCar, then action A_assignAssessor has two edge codesDS.4 and IC.1, and action A_sendNewRepairCost has two edge codes DS.5 and IC.3. 
A commitment graph is a directed graph consisting of a set of vertices corresponding to all roles $\mathbb{R}$, a set of edges corresponding to actions and their codes, and commitment orders .

Definition 8 Let $\mathbb{A}$ be a set of actions, $a \in \mathbb{A}, \mathbb{M}$ be a set of commitments, $m \in \mathbb{M}$, and $X=\{1,2, \ldots\}$, a sequence function $f_{\text {position }}(a, m)$, an edge is specified as a relation from $\mathbb{A} \times \mathbb{M} \times X$ :

$$
\text { edge }=\bigcup_{\forall m, a \in m}\left\{\left(a, m, f_{\text {position }}(a, m)\right): a \in \mathbb{A}, m \in \mathbb{M}, f_{\text {position }}(a, m) \in X\right\}
$$

a set of all edges is:

$$
\mathbb{E}=\bigcup_{\forall a \in \mathbb{A}}\{e d g e\}
$$

Definition 9 Let $\mathbb{M}$ is a set of commitments. A commitment occurrence order is specified as a relation from $\mathbb{M} \times \mathbb{M}$ :

$$
\text { order_commitment }=\left\{\left(m_{1} \cdot m_{2}\right): m_{1}, m_{2} \in \mathbb{M}\right\}
$$

If $m_{1} \cdot m_{2}$ is a commitment order, we inferpret it as: commitment $m_{2}$ is only active when commitment $m_{1}$ has been finished, i.e. only after the actions of $m_{1}$ has occurred in the past, the commitment $m_{2}$ can be inccoporated to trace back contract violations.

A set of commitment orders lists all relationships which a commitment occurs before than another commitment, it is specified as:

$$
\mathbb{O}=\bigcup_{\forall x \in \mathcal{P}}\left\{\left(m_{1} \cdot m_{2}\right)\right\}
$$

For the car insurance case, examples of the commitment orders inducively presented as following:

$$
\begin{aligned}
& \mathbb{O}=\left\{C_{\text {_p phoneService }} \cdot C_{\text {_repairService }} C_{\text {_ }} \text { phoneService } \cdot C_{\text {_d dailyService }}\right. \\
& \text { C_phoneService · C_claimForm, C_phoneService } \cdot \text { C_inspectCar, } \\
& \text { C_repairService - C_payRepairCost, C_dailyService - C_payRepairCost, } \\
& \text { C_inspectCar · C_payRepairCost, C_claimForm · C_payRepairCost }\}
\end{aligned}
$$

After we specify commitment graph notes, edges, and commitment occurrence orders, the commitment graph can be specified as following:

Definition 10 Let $\mathbb{R}$ be a set of nodes, $\mathbb{E}$ be a set of edges, and $\mathbb{O}$ be a set of commitment order list. The commitment graph can be specified as:

$$
G=(\mathbb{R}, \mathbb{E}, \mathbb{O})
$$


When an edge has more than one edge code, it means this action is involved in different commitments. The edge codes present a complicated relationship among commitments. A commitment graph is a visual tool to show commitment relationships in a complex multi-party contract, it assists in locating the responsible parties for violations.

Using commitment graphs, action occurrence is based on the commitment order and the sequence number with in a commitment. Some actions with multiabbreviation show that they involve in different commitments.

Algorithm for guard maintenance. Executing guards is a well-known operation with agent systems monitoring [27. When an action $a$ is attempted, its guard is evaluated, evaluation usually means checking if the guard evaluates to $\top$. Based on section 3.1 contract constraints and guards of contract constraints, we know actions can occur, can never occur, or have not yet occurred. If $a$ 's guard is satisfied, $a$ is executed; if it is $0, a$ is rejected; else the partner of perform action $a$ is made to wait. Whenever $a$ occurs, a notification is sent to each pertinent partner who performs action $b$, whose guards are updated accordingly. If $b$ 's guard becomes $T, b$ is allowed; if it becomes $0, b$ is rejected; otherwise, the partner perform action $b$ is made to wait some more and so on. In this algorithm, $\mathcal{A}$ is a set of all actions; $\Gamma=\{a, \bar{a}: a \in \mathcal{A}\}$ is set of actions and their complement, $\mathrm{U}$ and $\mathrm{V}$ are the temp set of actions and their complement, guard $[u]$ and guard $[v]$ are the temp storages of guards. We dynamically update our guards using Algorithm 1.Using the guard of the contract constraint scheme 1, if $a$

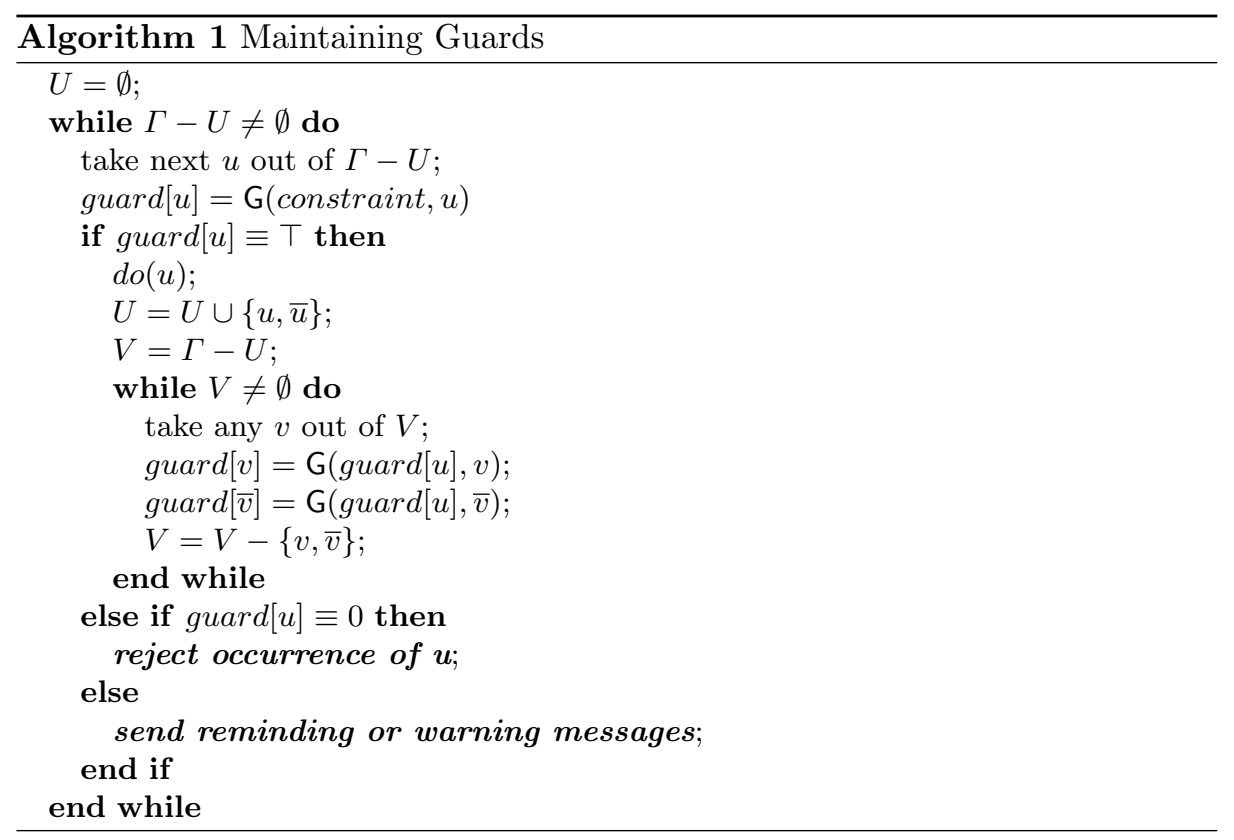


is attempted and $b$ has not already happened, $a$ 's guard evaluates to $\top$, consequently, $a$ is allowed and a notification $\square a$ is send. Upon receiption of this notification, $b$ 's guard is simplified from $\square a$ to $\top$. Now if $b$ is attempted, it can happen immediately. If $b$ is attempted first, it must wait because its guard is $\square$ a. Sometime later if $\square a$ received, which simplifies its guard to $T$.

The maintaining guard algorithm shows how to use guards to dynamically monitor business processes. It makes it possible to send the pertinent reminding message or warning message during the fulfillment of the contract.

Pro-active detection. In our approach, guards of contract constraints are used for proactive monitoring purposes. We will explain how to use guards and deadline of action together to trigger reactive module. The pro-active detection algorithm is specified in Algorithm 2, $w$ denotes a contract constraint scheme, $a, b$ are actions, $a, b \in w$; a.t indicates the deadline of action $a$; we use Petri Net to overview a business process [41] 13] 20] [19], the places correspond to states or each party and transitions correspond to actions of different parties, $M_{0}$ be a initial marking of Petri Net.

When an action is attempted, if the guard of the action evaluates to $T$, this action can occur immediately, the rest of actions will be reminded and their guards will be updated; let $f_{w}(a)$ be a function that gives warning time of action $a$. If $f_{w}(a)$ has passed and the action has not occurred yet, the warning module will be triggered and a reminding message will be send to parties involved. The time boundary between triggering reminding module and triggering warning module can be changed in different application.

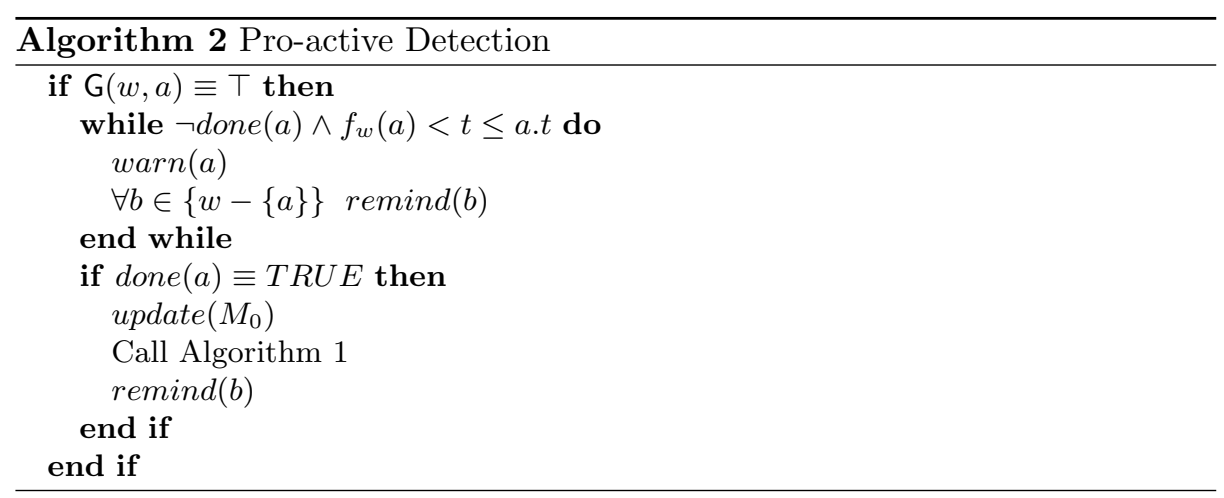

In above sections, we explain our monitoring approach which include a monitorable contract model and a dynamic monitoring mechanism. Using our proactive detection approach, the monitorability of contracts can be improved, avoidance and anticipation of violations are important features for our monitoring system. In next sections, the related work will discussed and compared. 


\section{Related Work}

Looking at the object-oriented constraints perspective, Bertrand Meyer [3] [2] refined the assertion based approach into the design by a contract method in the Eiffel language. The basic idea is that a component and its clients have a contract with each other. The client guarantees certain preconditions before calling a method, the component guarantees certain postconditions after the call. If the pre-and postconditions are included in a form that the compiler can check, then any violation of the contract between caller and component can be detected immediately. The prime focus of the approach is to deliver reliable software, and as such does not include pro-active monitoring.

Business process flow languages, like IBM's WSFL [9] and BPEL4WS [5], Microsoft's XLANG [39], provide a comprehensive structure for describing a business process in detail and there are also the obvious place to describe assertions. BPEL4WS focuses on non functional or procedural contract. Neither provides any explicit support for the "business contract" level of abstraction.

Various authors have proposed electronic contract models or languages based on different views. Kimbrough and Moore formalize and extend speech act theory [18 [33] 34] as Formal Language for Business Communication (FLBC) 37] [38], and apply it to deontic reasoning [36] and business messaging [37] 38]. Deontic logic [14] [42] based contract models [12] 44] [26] [32] [43] describe obligations, permissions, and forbiddances for finishing a business process, which work in an extremely ideal process. CrossFlow 16 and E-ADOME[15] use contracts for inter-organizational workflow process integration. Contracts in CrossFlow and E-ADOME describe the agreed workflow interfaces as activities and transitions, based on WfMC's WPDL (Workflow Process Definition Language). Contracts also specify what data objects in the remote workflow are readable or updateable. They are a side effect of business automations, and as for now does not have pro-active monitoring.

Other authors have added monitoring support to electronic contracts. Business Contract Architecture (BCA) 24 45 does not provide generic monitoring facilities, expecting each application to develop its own monitoring code to detect and signal non-conformance to the contract monitor; contract enforcement is limited to either signaling violation and or preventing the non-performing party from from entering further contracts. Daniel Reeves et al's declarative language for negotiating executable contracts can facilitate modification during negotiation [35] and include prioritized conflict handling features. However, it cannot be expected that all possible conflict scenarios throughout all business processes are recognized completely before the contract fulfillment stage. In SeCo (secure electronic contracts) [11, the monitoring services allow events to be triggered according to the current state of the contract and informs an enforcement service to initiate an enforcement activity. They only look at the negotiation stage. Daskalopulu et al. use subjective logic [40], which addresses a problem of a measurable belief, to realize evidence-based contract monitoring [7] [8] in the contract fulfillment stage. Unfortunately the monitoring is 
not pro-active. Milosevic et al. apply subjective logic to contract enforcement [25], but do not look at monitoring.

In existing e-commerce frameworks, the XML-based Trading Partner Agreement Markup Language (tpaML) from IBM research [6] 'trading partner agreement' are used as 'contract'. tpaML is now pursued under the OASIS Collaboration Protocol Profile (CPP) and Agreement (CPA) specifications [10]. tpaML and CPP/CPA capture the interoperation parameters (e.g. message formats, communication protocols, etc.), there are no provision for fulfillment monitoring. Microsoft's BizTalk has auditing and optional document mining function as well [23]. Their goals are to support recovering from failures, instead of prevention. Consequently, the development of contract models for contract fulfillment monitoring is relatively unexplored. Our approach provides a pro-active detection of imminent contract violations. Now we answer three questions which we address in introduction. Using guards of contract constraints and the guard maintenance algorithem, we answer question 1 to trace the current state of contract execution and to show the actions we can expect from a partner in the near future. Using the algorithm for pro-active detection, we answer question 2 to try to prevent contract violations by sending reminding and warning messages before. Using a commitment graph, we answer question 3 to show partners relevant to the contract violation.

\section{Conclusions}

This paper proposes an approach to formalize electronic contracts into a set of representations that enable automatic monitoring. The execution of a contract is seen as a stream of actions that happen over time. A paper contract is mapped into two parts. The first parts are the so-called contract constraints. These conditions are tested on their validity during contract execution. The second part of the contract are the commitments which are essentially guarantees by one partner to another partner that some action sequence will occur. The commitments are used to trace back a violation of a contract constraint to the partner who has made a matching commitment.

Apart from this main result, we see further achievements of our model that enhance its suitability for electronic contract executions. Imminent contract violations may be forecasted by checking the state of the so-called guard expressions ahead of the formal deadline of an expected action. This feature allows pro-active use of formal contract representations in order to avoid real violations.

Our execution model is compatible with workflow engines for distributed execution of multi-partner contracts. The action sequences can be seen as the log of a petri net machine which controls the execution of the contract. However, we do not require such an execution engine as long as a sequence of actions is generated for monitoring. Using a workflow engine would have the advantage that the checks for guards can be integrated into the proper workflow at the right place so that a partner no longer has to decide when to execute a check for an imminent or real contract violation. 
When contracts are automatically monitored, both the likelihood of violation decreases (as partners can be alerted in advance of a real violation) and opportunities for compensating violations are created. Rather than passing a violation case to a legal law suit, the failing partner can be forced to commit to a compensation that creates value for all partners. Without automatic monitoring, the detection of compensation opportunities is simply too costly to justify complete monitoring.

Further research has to be undertaken in the area of quality safeguards in electronic contracts. Lack of trust between partners may be dealt with by introducing a trusted third party which sub-divides actions into parts that are then irrevocable or provide monitoring services. An electronic contract can be analyzed prior to its execution in order to avoid incomplete commitment structures. Specifically, one may verify whether any violation of a contract constraint can be traced back to a commitment, i.e. a partner who is responsible for the violation. A prototype for algorithms 1 and 2 has been developed in prolog. We plan to test the prototype with action streams generated by a commercial execution environment for web services e.g. IBM WebSphere [4]. The test shall show in how far the message types provided there provide suitable action sequences needed for contract monitoring.

\section{References}

1. Abrahams A. An asynchronous rule-based approach for business process automation using obligations. the 3rd ACM SIGPLAN Workshop on Rule-Based Programming (RULE'02), Pittsburgh, USA, 2002.

2. Meyer B. Building bug-free oo software: An introduction to design by contract. http://www.eiffel.com/doc/manuals/technology/contract/.

3. Meyer B. Object-Oriented Software Construction $2^{\text {nd }}$ Edition. Prentice Hall, 1997.

4. IBM Corporation. Websphere. 2002. http://www-3.ibm.com/software/info1/websphere/index.jsp.

5. Klein J. Leymann F. Roller D. Thatte S. Curbera F., Goland Y. and Weerawarana S. Business process execution language for web services.

6. Dias D.M. Parr F.N. Kearney R. Sachs M.W. Lau T.C. Dan A., Nguyen T.N. and Shaikh H.H. Business-to-business integration with tpaml and a business-tobusiness protocol framework. Springer-Verlag, Lecture Notes in AI, 2000.

7. Dimitrako T. Daskalopulu A. and Maibaum T. Evidence-based electronic contract performance monitoring. INFORMS Journal of Group Decision and Negotiation, Special Issue: formal Modeling of Electronic Commerce, 2002.

8. Dimitrakos T. Daskalopulu A. and Maibaum T. S. E. E-contract fulfillment and agents' attitudes. Proceedings ERCIM WG E-Commerce Workshop on The Role of Trust in e-Business, 2001.

9. Leymann F. Web service flow language.

10. Organization for the Advancement of Structured Information Standards (OASIS). Oasis ebxml collaboration-protocol profile and agreement specification version 2.0. 2002.

11. Schopp B Greunz M and Stanoevska-Slabeva K. Supporting market transactions through xml contracting containers. Proceedings of the Sixth Americas Conference on Information Systems (AMCIS 2000). Long Beach, CA, 2000. 
12. Weigand $\mathrm{H}$. and $\mathrm{Xu}$ L. Contracts in e-commerce. 9th IFIP 2.6 Working Conference on Database Semantic Issues in E-Commerce Systems (DS-9), 2001.

13. Desel J. and Esparza J. Free Choice Petri Nets. Cambridge University Press, 1995.

14. Meyer J. and Wieringa R. Deontic Logic in Computer Science: Normative System Specification. John Wiley and Sons, 1993.

15. Chiu D. Kafeza E. and Kafeza I. View-based contracts in an e-service crossorganizational workflow environment. Proceedings of the second International Workshop on Technologies for E-Service(TES'01), 2001.

16. Grefen P Koetsier M. and Vonk. Cross-organisational/workflow: Crossflow esprite/28635 contract model, deliverable d4b. 1999.

17. Cohen P Kumar, S. Towards a fault-tolerant multi-agent system architecture. In Proceedings of The Fourth International Conference on Autonomous Agents (Agents 2000),ACM Press, pp. 459-466., 2000.

18. Austin J. L. How to do things with words. 2nd Edition. Oxford University Press, 1976.

19. Xu L. Car insurance case. Research Paper, Tilburg University.

20. Xu L. Agent-based monitorable contract. Research Paper, Tilburg University, 2002.

21. Xu L. and Jeusfeld M. A. A concept for monitoring of electronic contracts, research paper, tilburg university. http://infolab.uvt.nl/pub/itrs/itrs010.pdf.

22. Venkatraman M. and Singh M. P. Verifying compliance with commitment protocols: Enabling open web-based multiagent systems. Autonomous Agents and Multi-Agent Systems. volume 2, number 3.

23. Andrews G.M.T. Beckman B. Klein J. Mital A. Mehta B., Levy M. Biztalk service 2000 business process orchestration. International Conference on Data Engineering (ICDE'02).

24. Bond A. Milosevic Z., Berry A. and Raymond K. Supporting business contracts in open distributed systems. 2nd International Workshop on Services in Distributed and Networked Environments,(SDNE'95) Whistler, Canada, 1995.

25. Dimitrakos T. Milosevic Z., Jøsang A and Patton M.A. Discretionary enforcement of electronic contracts. Proceedings of EDOC'2000. IEEE Comp. Soc. Press, 2002.

26. Sierra C. Norman T.J. and Jennings N.R. Rights and commitments in multiagent agreements. Proceedings of the 3rd International Conference on Multi-Agent Systems (ICMAS-98). Paris, France, 1998.

27. Singh M. P. A customizable coordination service for autonomous agents. International Workshop on Agent Theories, Architectures, and Languages (ATAL).

28. Singh M. P. Developing formal specifications to coordinate heterogeneous autonomous agents. Proceedings of the International Conference on Multiagent Systems (ICMAS).

29. Singh M. P. Synthesizing coordination requirements for heterogeneous autonomous agents. Autonomous Agents and Multi-Agent Systems. volume 3, number 2.

30. CrossFlow Project. Insurance requirements. CrossFlow consortium.

31. Cohen P. R. and Levesque H. J. Communicative actions for artificial agents. In Victor Lesser and Les Gasser, editors, Proceedings of the First International Conference on Multi-Agent Systems (ICMAS'95), pages 65-72, San Francisco, CA, USA, 1995. The MIT Press: Cambridge, MA, USA.

32. Lee R. Towards open electronic contracting. Electronic Markets, Vol. 8, No. 3, $10 / 98$.

33. Searle J. R. Speech acts: An essay in the philosophy of language. Cambridge University Press, 1969. 
34. Searle J. R. and Vanderveken D. Foundations of Illocutionary Logic. Cambridge University Press, 1985.

35. Wellman M. P Reeves D. M., Grosof B. N. and Chan H. Toward a declarative language for negotiating executable contracts. In Proceedings of the AAAI-99 Workshop on Artificial Intelligence in Electronic Commerce (AIEC-99), Menlo Park, CA, USA, 1999.

36. Kimbrough S. Reasoning about the objects of attitudes and operators: Towards a disquotation theory for the representation of prepositional content. Eight International Conference on Artificial Intelligence and the Law (ICAIL 2001), 2001.

37. Kimbrough S. and Moore S. On automated message processing in electronic commerce and work support systems: Speech act theory and expressive felicity. ACM Transactions on Information Systems. 15(4). ACM Press. New York, NY. pp. 321367, 1997.

38. Moore S. Kqml and flbc: Contrasting agent communication languages. International Journal of Electronic Commerce 5(1), 2000.

39. Thatte S. Xlang web services for business process design.

40. Jøsang A. A logic for uncertain probabilities. International Journal of Uncertainty, Fuzziness and Knowledge-Based Systems. 9(3), pp. 279-311, 2001.

41. Reisig V. A primer in Petri net design. Springer-Verlag, 1992.

42. von Wright G. Deontic logic. Mind, 1951.

43. Verharen E. Weigand H., Dignum F. Dynamic business models as a basis for interoperable transaction design. Information Systems, 1997.

44. Tan Y.H. and Thoen W. A logical model of directed obligations and permissions to support electronic contracting in electronic commerce. International Journal of Electronic Commerce (IJEC). 3(2). pp. 87-104.

45. Milosevic Z. Enterprise Aspects of Open Distributed Systems. PhD Thesis, pp. 154-248. Department of Computer Science, University of Queensland, 1995. 